Osamu Miyoshi - Satoshi Hayashi • Masahiro Fujimoto

Hiroaki Tomita • Masakazu Sohda • Norio Niikawa

\title{
Maternal uniparental disomy for chromosome 14 in a boy with intrauterine growth retardation
}

\begin{abstract}
Maternal uniparental disomy (UPD) for chromosome 14 [upd(14)mat] may cause a characteristic phenotype with growth and developmental deficiency and precocious puberty. We report the case of a Japanese infant with an isochromosome $14[\mathrm{i}(14 \mathrm{q})]$ and intrauterine growth retardation (IUGR). The infant is one of triplets comprising a boy (the patient) and two karyotypically normal girls. We analyzed parent-child transmission modes of alleles on the i(14q) at 17 CA-repeat marker loci along the entire length of chromosome 14. Genotypes at 4 proximal and 5 distal loci on the $\mathrm{i}(14 \mathrm{q})$ were consistent with maternal isodisomy, whereas those at an intervening region indicated maternal heterodisomy. Thus, the derivative chromosome 14 had arisen through a translocation between maternal homologous chromosomes $14[\mathrm{t}(14 ; 14)(\mathrm{p} 10 ; \mathrm{q} 10)]$ after at least two crossing-over events at the first meiosis. This result also suggests that there must be maternally imprinted gene(s) on $14 q$, and that loss of the functionally active, paternally derived allele in the same locus may lead to IUGR. Alternatively, IUGR may be an autosomal recessive trait. In the latter case, the mother would be a heterozygote and the putative disease locus would be either at the most proximal or most distal region of $14 \mathrm{q}$.
\end{abstract}

Key words Chromosome $14 \cdot$ Maternal uniparental disomy - Genomic imprinting · Autosomal recessive trait · Intrauterine growth retardation

O. Miyoshi $(\bowtie) \cdot$ M. Fujimoto $\cdot$ H. Tomita $\cdot$ N. Niikawa Department of Human Genetics, Nagasaki University School of Medicine, Sakamoto 1-12-4, Nagasaki 852-8523, Japan

Tel. +81-95-849-7120; Fax +81-95-849-7121

e-mail: f1119@cc.nagasaki-u.ac.jp,

S. Hayashi

Department of Obstetrics and Gynecology, Hiroshima Prefectural Hospital, Hiroshima, Japan

M. Sohda

Kyushu Medical Science, Nagasaki Laboratory, Nagasaki, Japan

\section{Introduction}

Uniparental disomy (UPD) occurs when both members of homologous chromosomes are derived from one parent. When the two homologs in the child are derived from the two corresponding homologs in one parent, it is called uniparental heterodisomy, whereas when both of the homologs originate from one chromosome in duplicate, it is uniparental isodisomy (Engel 1980). The two types of UPD may contribute to an abnormal phenotype, if a gene on the UPD chromosome is functionally inactive through a genomic imprinting mechanism (Nicholls 1993; Cattanach 1991; Ledbetter and Engel 1995) or through a mutation that is responsible for a recessive trait (Spence et al. 1988; Voss et al. 1989; Pentao et al. 1992; Sulisalo et al. 1997). Thus, UPD can provide evidence for the existence of an imprinted gene in the human genome, or information for mapping a recessive disease. To our knowledge, there have been 11 cases of maternal UPD for chromosome 14 [upd(14)mat] (Temple et al. 1991; Pentao et al. 1992; Antonarakis et al. 1993; Healey et al. 1994; Morichon-Delvallez et al. 1994; Robinson et al. 1994; Sirchia et al. 1994; Papenhausen et al. 1995; Tomkins et al. 1996; Coviello et al. 1995; Splitt and Goodship 1997). The majority of individuals with upd(14)mat had characteristic clinical manifestations such as prenatal and/or postnatal growth deficiency, developmental retardation, and precocious puberty (Hurst and $\mathrm{McVean}$ 1997). Here, we report on an infant with intrauterine growth retardation (IUGR) and a 45,XY,i(14q) karyotype, another case of upd(14)mat.

\section{Materials and methods}

Patient

The patient, a 9-month-old Japanese boy, is one of triplets born to unrelated, healthy parents. The ages of his mother and father at the time of delivery were 32 years and 35 years, respectively. Because four previous pregnancies in 


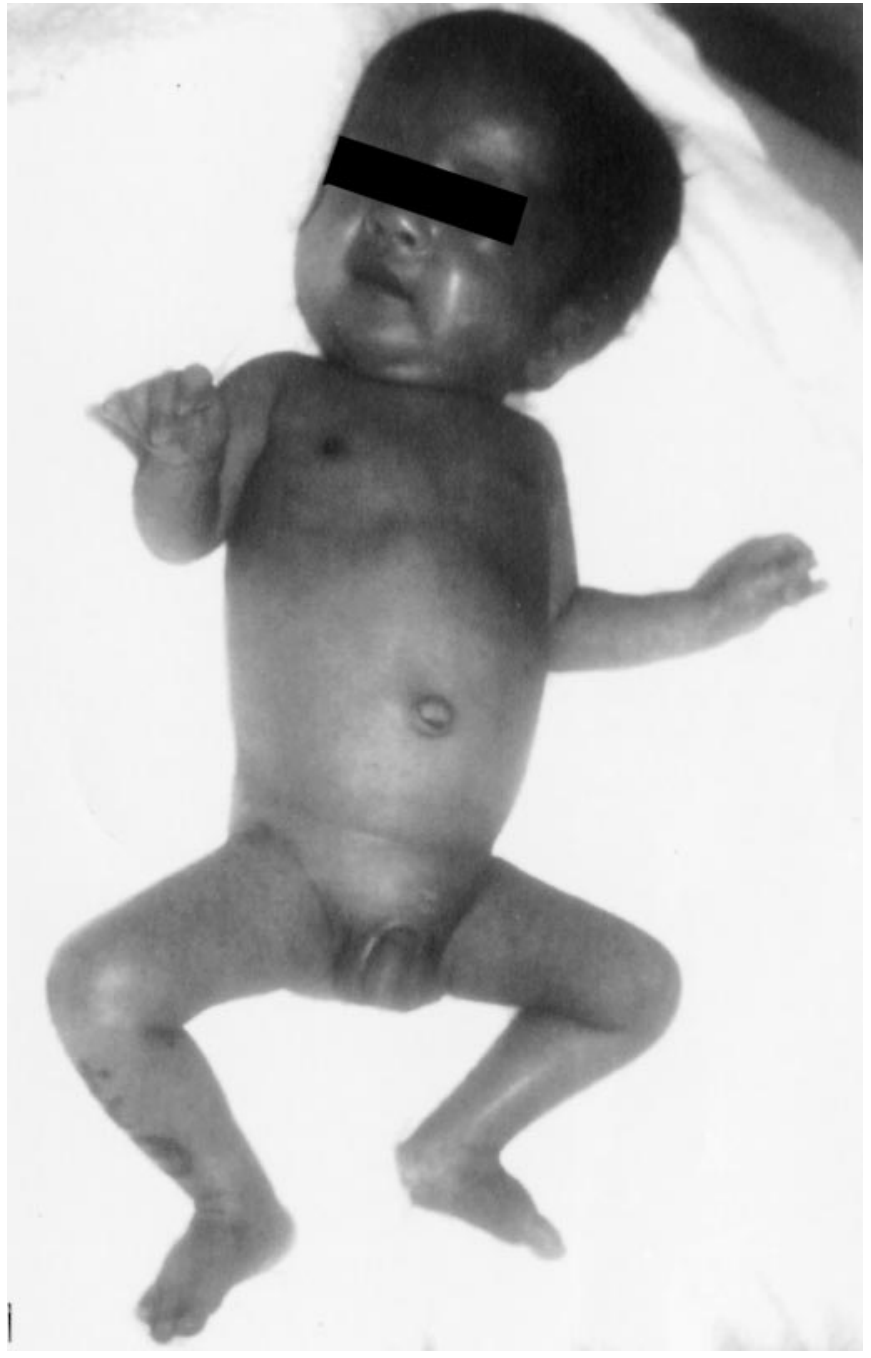

Fig. 1. Patient at age 4 months

the mother ended in spontaneous abortions, the mother received both immunotherapy with a hypodermic injection of paternal lymphocytes and hormonal therapy with a combination of human menopausal gonadotropin (hMG) and human chorionic gonadotropin (hCG). The other members of the triplets, two girls, were healthy and both weighed $1110 \mathrm{~g}$, appropriate for their date of birth. There was no other family history of any multiple congenital anomaly or mental retardation syndrome.

At 29 gestational weeks, the boy was delivered by Caesarean section, with a weight of $634 \mathrm{~g}$ (small for the date), a length of $31 \mathrm{~cm}$, and a head circumference of $25.6 \mathrm{~cm}$. The patient was hypotonic, with failure to thrive, and had respiratory distress that required immediate intubation and assisted-ventilation. Physical examination revealed the following abnormalities: dolicocephaly with frontal bossing, low-set ears, short neck, small hands and feet, overlapping fingers (Fig. 1), and patent ductus arteriosus (PDA). A skeletal survey showed no abnormality. Medical evaluation at age 4 months demonstrated a low serum IgG level (159 mg/dl), but electroencephalography, brain computed tomography, and magnetic resonance im-

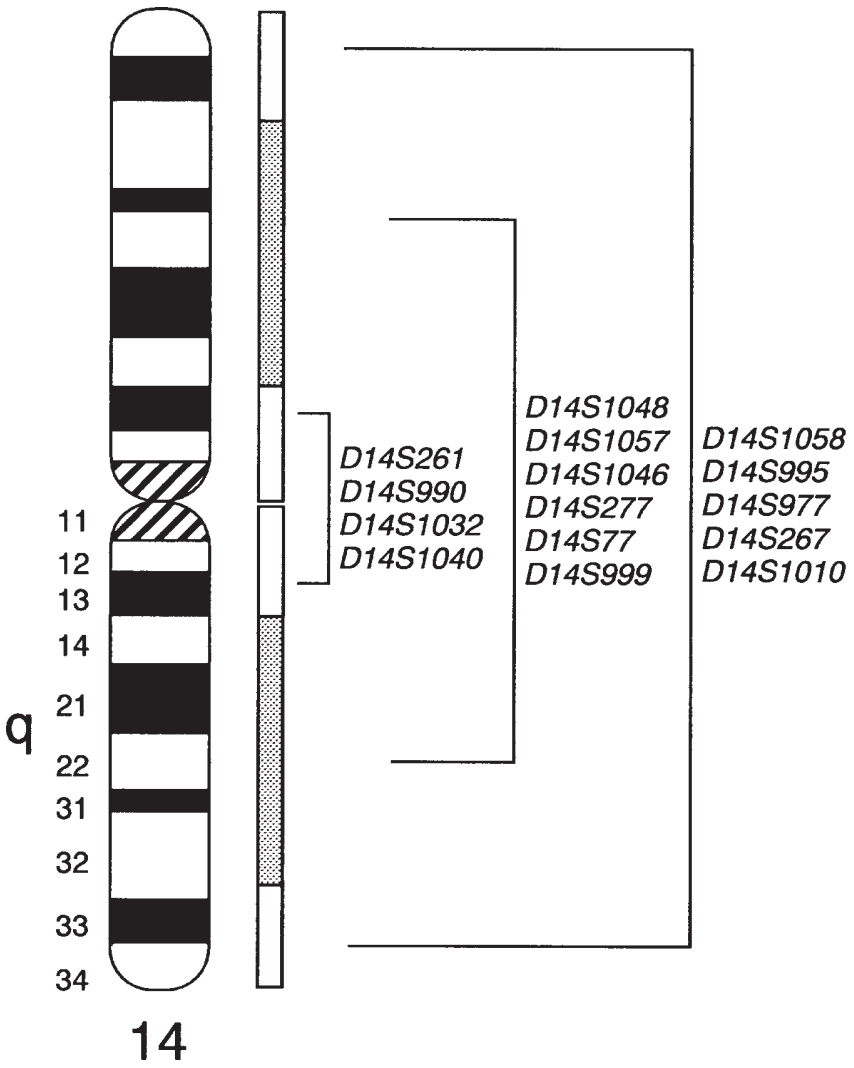

Fig. 2. Idiogram of the patient's $\mathrm{t}(14 \mathrm{q} ; 14 \mathrm{q})$ chromosome. Open and shaded boxes depict isodisomic and heterodisomic regions, respectively

aging were all normal. He was discharged after 137 days. Examination at 259 days revealed growth and developmental retardation, with a length of $59.3 \mathrm{~cm}$, weight of $4210 \mathrm{~g}$, and head circumference of $41.0 \mathrm{~cm}$. The serum IgG level became normal $(400 \mathrm{mg} / \mathrm{dl})$. Chromosome analysis on peripheral blood lymphocytes from the patient revealed a $45, \mathrm{XY}, \mathrm{i}(14)(\mathrm{q} 10)$ or $45, \mathrm{XY}, \mathrm{t}(14 ; 14)(\mathrm{p} 10 ; \mathrm{q} 10)$ karyotype (Fig. 2). Karyotypes of the other two triplet-members and the parents were normal.

\section{Triplet zygosity and genotype analysis}

To ascertain the zygosity of the triplets, chromosome analysis was performed using quinacrine fluorescence Q (QFQ)banded heteromorphisms as chromosome markers, as described previously (Abe et al. 1996). Next, a DNA marker study was done to determine their genotypes. Genomic DNA was extracted from peripheral blood leukocytes of the patient, his sisters, and his parents by the use of a DNA-extraction kit (Wako Chemical, Tokyo, Japan), according to the manufacturer's directions. The origin of formation of the isochromosome in the patient was studied by tracing parent-child transmission of polymorphic alleles on chromosome 14 . We chose 17 microsatellite (CA-repeat) markers that are mapped along the long-arm chromosome 14 from the published genetic maps (Cuticchia et al. 1995; Dib et al. 1996) (Table 1). In addition, microsatellite markers on chromosomes 10,13 , and 18 were used to con- 
Table 1 Zygosity of patient's sisters

\begin{tabular}{lllll}
\hline \multirow{2}{*}{ Chromosome } & \multicolumn{4}{l}{ Heteromorphic patterns } \\
\cline { 2 - 5 } & Father & Mother & Sister 1 & Sister 2 \\
\hline 3 & a, b & b, b & b, b & a, b \\
4 & b, b & a, b & b, b & a, b \\
13 & a, b & b, b & a, b & a, b \\
14 & a, b & b, c & a, c & a, c \\
15 & a, b & b, b & a, b & b, b \\
21 & a, b & a, c & a, a & a, b \\
22 & a, b & a, c & a, a & b, c \\
\hline
\end{tabular}

Table 2 Genotypes at CA-repeat marker loci in the patient and his parents

\begin{tabular}{lllll}
\hline \multirow{2}{*}{ Probes } & \multirow{2}{*}{ Locations } & \multicolumn{3}{l}{ Genotypes (type of UPD) } \\
\cline { 3 - 5 } & & Father & Mother & Patient \\
\hline D14S261 & 14q11 & 3,3 & 2,1 & $2,2(\mathrm{I})$ \\
D14S990 & 14q11.1-q12 & 2,4 & 1,3 & $1,1(\mathrm{I})$ \\
D14S1032 & 14q11.1-q13 & 1,2 & 2,3 & $3,3(\mathrm{I})$ \\
D14S1040 & 14q12-q13 & 1,2 & 1,3 & $3,3(\mathrm{I})$ \\
D14S1049 & 14q12-q13 & 2,3 & 1,2 & $2,2(\mathrm{U})$ \\
D14S1048 & 14q12-q13 & 2,3 & 1,4 & $1,4(\mathrm{H})$ \\
D14S1057 & 14q13-q21 & 2,3 & 1,1 & $1,1(\mathrm{H})$ \\
D14S1046 & 14q23 & 1,2 & 3,4 & $3,4(\mathrm{H})$ \\
D14S277 & 14q23-q24.3 & 1,2 & 3,4 & $3,4(\mathrm{H})$ \\
D14S77 & 14q24 & 2,3 & 1,4 & $1,4(\mathrm{H})$ \\
D14S999 & 14q24.3-q31 & 2,4 & 1,3 & $1,3(\mathrm{H})$ \\
D14S61 & 14q24.3-q31 & 1,2 & 1,3 & $1,3(\mathrm{U})$ \\
D14S1058 & 14q24.3 & 2,2 & 1,1 & $1,1(\mathrm{I})$ \\
D14S995 & 14q24.3-q31 & 2,4 & 1,3 & $3,3(\mathrm{I})$ \\
D14S977 & 14q31 & 2,3 & 1,1 & $1,1(\mathrm{I})$ \\
D14S267 & 14q32.1-q32.2 & 2,3 & 1,2 & $1,1(\mathrm{I})$ \\
D14S1010 & 14q32.1-q32.3 & 2,4 & 1,3 & $1,1(\mathrm{I})$ \\
D10S196 & 10q11.2-q21.2 & 2,4 & 1,3 & 1,4 \\
D13S1253 & 13q13-q14.2 & 1,4 & 2,3 & 3,4 \\
D18S57 & 18q12.2-q12.3 & 2,3 & 1,1 & 1,3 \\
\hline UPD
\end{tabular}

UPD, uniparental disomy; I, isodisomy; $\mathrm{H}$, heterodisomy; $\mathrm{U}$, uninformative.

firm paternity. We generated Cy-5 labeled primer DNAs and their respective unlabeled reverse primers for polymerase chain reaction (PCR) amplification of marker DNAs, according to Dib et al. (1996). The PCR was cycled 30 times under the following conditions: denaturation at $95^{\circ} \mathrm{C}$ for $30 \mathrm{~s}$, annealing at $55^{\circ} \mathrm{C}$ for $30 \mathrm{~s}$, and extension at $72^{\circ} \mathrm{C}$ for $30 \mathrm{~s}$ in a mixture containing $50 \mathrm{mM} \mathrm{KCl}$, $20 \mathrm{mM}$ Tris- $\mathrm{HCl}(\mathrm{pH} 8.5), 1.5 \mathrm{mM} \mathrm{MgCl}, 200 \mathrm{mM}$ each of dNTPs, and 0.5 U AmpliTaq (Perkin Elmer, Norwalk, CT, USA). PCR products were sequenced in an automated sequencer (ALFexpress DNA sequencer, Pharmacia Biotech, Uppsala, Sweden), electrophoretic patterns were analyzed with a software package, Fragment Manager (Pharmacia Biotech), and genotypes of the family members were determined.

\section{Results}

Karyotyping as well as QFQ heteromorphism analysis demonstrated that the triplets are trizygotic (Table 1). CA- repeat analysis on chromosome 14 showed that the patient lacks any of the paternal alleles but has inherited two copies of the maternal alleles at all loci examined, indicating maternal UPD for chromosome 14 (Table 2). The patient has two copies of one-maternal-allele both at 4 centromeric loci (D14S261,D14S990,D14S1032, and D14S1040) and at 5 telomeric loci (D14S1058, D14S995, D14S977, D14S267, and D14S1010) (Table 2, Fig. 2). On the other hand, he inherited both of the maternal alleles at the remaining 6 intermediate loci (D14S1048, D14S1057, D14S1046, D14S277, D14S77, and D14S999). The other 2 markers (D14S1049 and D14S61) were uninformative, but their transmission modes were not inconsistent with maternal UPD. These results indicated that both centromeric and telomeric regions of the chromosome 14 are isodisomic, whereas the other region is heterodisomic, i.e., segmental UPD. Biparental inheritance of chromosomes 10,13, and 18 was confirmed using the D10S196, D13S1253, and D18S57 markers.

\section{Discussion}

Constitutional euploid UPD may arise through one of the following mechanisms (Engel 1980; Spence et al. 1988): (1) fusion of a nullisomic gamete with a gamete disomic for the same chromosome (gametic complementation); (2) loss of one member of trisomic chromosomes in a zygote at an early mitotic division (trisomy rescue); or (3) duplication of a monosomic chromosome in a zygote (monosomy duplication). The "gametic complementation" mechanism may result in partial (or segmental) UPD, depending on meiotic cross-overs, and the "trisomy rescue" may reconstitute a normal zygote in $2 / 3$ cases and complete or segmental UPD in $1 / 3$, whereas the "monosomy duplication" mechanism leads to complete isodisomy, i.e., homodisomy. Postzygotic loss of a chromosome and simultaneous duplication of the remaining counterpart chromosome may also result in UPD, usually leading to mosaicism, unless the event occurs at the first mitotic division (Slatter et al. 1994).

UPD with a Robertsonian or whole-arm translocation between homologous chromosomes or an isochromosome without its normal chromosome member, as in our patient, can arise through any one of these three different mechanisms, with the exception of a chromosome rearrangement event occurring before or after conception. Our polymorphic marker study on the translocated chromosome 14 in the patient demonstrated maternal UPD with isodisomy for the centromeric and telomeric regions and heterodisomy for the middle region of the chromosome. This indicates that meiotic recombinations had occurred between the maternal homologs 14, and thus favors either (1) or (2) as the mechanism. The observed nonmosaicism in our patient does not support a postzygotic event. It remains to be determined whether the immunotherapy and/or the hormonal therapy given to the mother may have induced UPD.

Individuals with maternal or paternal UPD often have unusual clinical manifestations. The manifestations may be 
Table 3 Clinical findings in patients reported with UPD for chromosome 14

\begin{tabular}{llll}
\hline & \multicolumn{2}{l}{ UPD for chromosome 14} & \\
\cline { 2 - 4 } Manifestations & Maternal (\%) & Paternal & Present case \\
\hline Intrauterine growth & $6 / 7(86)$ & $0 / 4$ & 1 \\
$\quad$ retardation (IUGR) & & & \\
Growth deficiency & $10 / 12(83)$ & $3 / 3$ & + \\
Neonatal hypotonia & $5 / 7(71)$ & $2 / 4$ & + \\
Hydrocephaly & $3 / 7(43)$ & $0 / 3$ & - \\
Cleft/high-arched plate & $3 / 7(43)$ & $1 / 4$ & - \\
Small hands and feet & $6 / 7(86)$ & $0 / 2$ & + \\
Scoliosis & $4 / 9(44)$ & $1 / 4$ & - \\
Mental retardation & $0 / 7(0)$ & $1 / 3$ & $?$ \\
Precocious puberty & $6 / 7(86)$ & $?$ & $?$ \\
Polyhydramnios & $0 / 7(0)$ & $3 / 4$ & - \\
Blepharophimosis & $3 / 3$ & - & \\
$\quad$ and/or short & & & \\
$\quad$ palpebral fissures & & - & \\
Protruding philtrum & $3 / 3$ & - & \\
Small ears & $3 / 3$ & - & \\
Small thorax with & $3 / 3$ & - & \\
$\quad$ abnormal ribs & & & \\
Gastric ulcer & $3 / 3$ &
\end{tabular}

${ }^{a}$ IUGR is judged by a birth-weight below the 10th percentile of values corresponding to gestational age. Data are from Temple et al. 1991; Pentao et al. 1992; Antonarakis et al. 1993; Healey et al. 1994; Robinson et al. 1994; Sirchia et al. 1994; Morichon-Delvallez et al. 1994; Papenhausen et al. 1995; Coviello et al. 1995; Tomkins et al. 1996; Splitt and Goodship 1997; Wang et al. 1991; Walter et al. 1996; Cotter et al. 1997.

explained by either genomic imprinting or homozygosity of a mutated gene. Maternal UPD for chromosome 14 [upd(14)mat] has been identified in 12 individuals including our patient (Temple et al. 1991; Pentao et al. 1992; Antonarakis et al. 1993; Healey et al. 1994; MorichonDelvallez et al. 1994; Robinson et al. 1994; Sirchia et al. 1994; Papenhausen et al. 1995; Tomkins et al. 1996; Coviello et al. 1995; Splitt and Goodship 1997). All but two of these individuals (Morichon-Delvallez et al. 1994; Papenhausen et al. 1995) had a common phenotype, including growth and developmental retardation, neonatal hypotonia, small hands and feet, and precocious puberty (Table 3). On the other hand, paternal UPD for chromosome 14 [upd(14)pat] is associated with different manifestations, such as polyhydramnios, minor craniofacial anomalies, small thorax with abnormal ribs, and gastric ulcer (Wang et al. 1991; Papenhausen et al. 1995; Walter et al. 1996; Cotter et al. 1997), although some other features seem to overlap with upd(14)mat (Table 3). Thus, the phenotype characteristic of maternal UPD is most likely due to a lack of active paternal allele(s) on maternally imprinted loci.

Similar phenomena have been observed in maternal and paternal UPD for chromosome 15 in Prader-Willi syndrome and in Angelman syndrome, respectively (Nicholls 1993; Niikawa 1996), and in paternal UPD for chromosome 11 in Wiedemann-Beckwith syndrome (Henri et al. 1994). Alternatively, IUGR (or growth retardation) and/or other manifestations, as in our patient, could be autosomal recessive traits. Examples include cystic fibrosis (Spence et al. 1988; Voss et al. 1989); cartilage hair hypoplasia, McKusick type (Sulisalo et al. 1997); and rod monochromacy (Pentao et al. 1992); in which patients had UPD of chromosome 7 , chromosome 9, and chromosome 14, respectively. In this case, the mother of our patient would be a heterozygote and the putative disease locus would be either at the most proximal or most distal region of 14q. However, this is less likely. The occurrence of growth retardation in 10 of the 12 reported patients with upd(14)mat is much more frequent than would be expected for an autosomal recessive trait, because the gene frequency for such a recessive trait would be expected to be relatively low. Furthermore, IUGR has never been reported in cases of upd(14)pat. Therefore, genomic imprinting is a likely mechanism for IUGR.

Acknowledgments We are grateful to Dr. Tadashi Kajii for his helpful suggestions on the work, Dr. Naoki Yokoyama for providing clinical information on the patient, and to Dr. Shinji Kondo and Mr. Naoki Harada for their technical assistance. This work was supported in part by a Grant-in-Aid for Scientific Research (No. 08307019) from the Ministry of Education, Science, Sport, and Culture of Japan.

\section{References}

Abe K, Harada N, Itoh T, Hirakawa O, Niikawa N (1996) Trisomy 13/ trisomy 18 mosaicism in an infant. Clin Genet 50: 300-303

Antonarakis SE, Blouin JL, Maher J, Avramopoulos D, Thomas G, Talbot CC Jr (1993) Maternal uniparental disomy for human chromosome 14 , due to loss of a chromosome 14 from somatic cells with $\mathrm{t}(13 ; 14)$ trisomy 14. Am J Hum Genet 52: 1145-1152

Cattanach BM (1991) Chromosomal imprinting and its significance for mammalian development. In: Davies KE, Tilghman SM (eds) Gene expression and its control. Cold Spring Harbor Laboratory Press, Cold Spring Harbor, New York, pp 41-71

Cotter PD, Kaffe S, McCurdy LD, Jhaveri M, Willner JP, Hirschhorn K (1997) Paternal uniparental disomy for chromosome 14. A case report and review. Am J Med Genet 70: 74-79

Coviello DA, Panucci E, Mantero MM, Perfumo C, Guelfi M, Borronco C, Dagna-Bricarelli F (1995) Maternal uniparental disomy for chromosome 14. Am J Hum Genet 57: 111A

Cuticchia AJ, Chipperfield MA, Foster PA (1995) Human gene mapping - a compendium. Johns Hopkins University Press, Baltimore, pp 838-844

Dib C, Fauré S, Fizames C, Samson D, Drout N, Vignal N, Millasseau P, Marc S, Hazan J, Seboun E, Lathrop M, Gyapay G, Morissette J, Weissenbach J (1996) A comprehensive genetic map of the human genome based on 5264 microsatellites. Nature 380: 152-154

Engel E (1980) A new genetic concept: uniparental disomy and its potential effect, isodisomy. Am J Med Genet 6: 137-143

Healey S, Powell F, Battersby M, Chenevix-Trench G, McGill J (1994) Distinct phenotype in maternal uniparental disomy of chromosome 14. Am J Med Genet 51: 147-149

Henry I, Puech A, Riesewijk A, Ahnine L, Mannens M, Beldjord C, Bitoun P, Tournade MF, Landrieu P, Junien C (1993) Somatic mosaicism for partial paternal isodisomy in Wiedemann-Beckwith syndrome: a post-fertilization event. Eur J Hum Genet 1: 19-29

Hurst LD, McVean GT (1997) Growth effects of uniparental disomies and the conflict theory of genomic imprinting. Trends Genet 13: 436443

Ledbetter DH, Engel E (1995) Uniparental disomy in humans: development of an imprinting map and its implications for prenatal diagnosis. Hum Mol Genet 4: 1757-1764

Morichon-Delvallez N, Segues B, Pinson MP, Bérubé D, Dommergues M, Aubry MC, Cessot F, Lyonnet S, Munnich A, Vekemans M (1994) Maternal uniparental disomy for chromosome 14 by secondary nondisjunction of an initial trisomy. Am J Hum Genet 55: A2224

Nicholls RD (1993) Genomic imprinting and uniparental disomy in Angelman and Prader-Willi syndromes: A review. Am J Med Genet 46: $16-25$ 
Niikawa N (1996) Genomic imprinting and its relevance to genetic diseases. Jpn J Hum Genet 41: 351-362

Papenhausen PR, Mueller OT, Johnson VP, Sutcliffe M, Diamond TM, Kousseff BG (1995) Uniparental disomy of chromosome 14 in two cases: An abnormal child and a normal adult. Am J Med Genet 59: 271-275

Pentao L, Lewis RA, Ledbetter DH, Patel PI, Lupski JR (1992) Maternal uniparental isodisomy of chromosome 14: Association with autosomal recessive rod monochromacy. Am J Hum Genet 50: 690-699

Robinson WP, Bernasconi F, Basaran S, Yuksel-Apak M, Neri G, Serville F, Balicek P, Haluza R, Farah LMS, Schinzel AA (1994) A somatic origin of homologous Robertsonian translocations and isochromosomes. Am J Hum Genet 54: 290-302

Sirchia SM, DeAdnreis C, Pariani S, Grimoldi MG, Molinari A, Buscaglia M, Simoni G (1994) Chromosome 14 maternal uniparental disomy in the euploid cell line of a fetus with mosaic $46, \mathrm{XX}$ 47,XX,+14 karyotype. Hum Genet 94: 355-358

Slatter RE, Elliott M, Welham K, Carrera M, Schofield PN, Barton DE, Maher ER (1994) Mosaic uniparental disomy in BeckwithWiedemann syndrome. J Med Genet 31: 749-753

Spence JE, Perciaccanate RG, Greig GM, Willard HF, Ledbetter DH, Hejtmancik JF, Pollack MS, O'Brien WE, Beaudet AL (1988) Uniparental disomy as a mechanism for human genetic disease. Am J Hum Genet 42: 217-226
Splitt MP, Goodship JA (1997) Another case of maternal uniparental disomy chromosome 14 syndrome. Am J Med Genet 72: 239-240

Sulisalo T, Makitie O, Sistonen P, Rodanpaa M, El-Rifai W, Ruuskanen O, de la Chapelle A, Kaitila I (1997) Uniparental disomy in cartilage-hair hypoplasia. Eur J Hum Genet 5: 35-42

Temple IK, Cockwell A, Hassold T, Pettay D, Jacobs P (1991) Maternal uniparental disomy for chromosome 14. J Med Genet 28: 511-514

Tomkins DJ, Roux AF, Waye J, Freeman VCP, Cox DW, Whelan DT (1996) Maternal uniparental isodisomy of human chromosome 14 associated with a paternal $\mathrm{t}(13 \mathrm{q} 14 \mathrm{q})$ and precocious puberty. Eur $\mathrm{J}$ Hum Genet 4: 153-159

Voss R, Ben-Simon E, Avital A, Godfrey S, Zlotogora J, Dagan J, Tikochinski Y, Hillel J (1989) Isodisomy of chromosome 7 in a patient with cystic fibrosis: Could uniparental disomy be common in humans? Am J Hum Genet 45: 373-380

Walter CA, Shaffer LG, Kaye CI, Huff RW, Ghidoni PD, McCaskill C, McFarland MB, Moore CM (1996) Short limb dwarfism and hypertrophic cardiomyopathy in a patient with paternal isodisomy 14:45,XY,idic(14)(p11). Am J Med Genet 65: 259-265

Wang JC, Passage MB, Yen PH, Shapiro LJ, Mohandas TK (1991) Uniparental heterodisomy for chromosome14 in a phenotypically abnormal familial balanced 13/14 Robertsonian translocation carrier. Am J Hum Genet 48: 1069-107 